\title{
Foam Behaviour of An Aqueous Solution of Piperazine- N-Methyldiethanolamine (MDEA) Blend as A Function of The Type of Impurities and Concentrations
}

\author{
Iwan Ratman $^{1}$, T.D. Kusworo ${ }^{2}$, and A.F. Ismail ${ }^{3}$ \\ ${ }^{I}$ Total SA, Strategy Business Development, LNG Departemnt, Tour Couple, La Defense, Paris, France \\ ${ }^{2}$ Department of Chemical Engineering, \\ University of Diponegoro, Jl. Prof. Sudharto SH No. 1, Semarang, Indonesia \\ ${ }^{3}$ Advanced Membrane Research Technology Center, \\ Faculty of Chemical and Natural Resources Engineering, \\ Universiti Teknologi Malaysia, 81310 UTM, Skudai, Johor Bahru, Malaysia
}

\begin{abstract}
This study focuses on the effect of impurities in the natural gas stream on the characteristic of foam behaviour in the blended piperazine and MDEA solution. Hydrocarbon liquids, Iron Sulphide, Sodium Chloride, Acetic Acid, Methanol and Polyethylene Glycol were used as the impurities. The results indicated that the type of impurities determined the foam formation of the amine solution. The concentration of piperazineMDEA blends also enhanced to the increasing of the foam height of blended piperazine-MDEA. Iron sulfide, hydrocarbon and sodium chloride are the impurities which apparently contributed to the high foaming tendency of the solutions. At the same concentration of the impurities, iron sulfide appeared as the most influential contaminant to the foam formation, which promoted the highest foamability in any concentrations of the blend piperazine-MDEA.
\end{abstract}

Keyword - piperazine-MDEA, foam behaviour, amine degradation.

\section{INTRODUCTION}

Natural, synthesis, and refinery of the raw gases contain acid gases such as $\mathrm{H}_{2} \mathrm{~S}$ and $\mathrm{CO}_{2}$. Removal of acid gas from gas mixtures is very important in natural gas processing, hydrogen purification, refinery off gases treatment and synthesis gas for ammonia and methanol making (Bhide et al., 1998). Acid gases must be removed from natural gas in order to: (a). increase the heating value of natural gas, (b). decrease the volume of gas transported in pipelines, (c). reduce corrosion during the transport and distribution of natural gas, and (d). prevent atmospheric pollution by $\mathrm{SO}_{2}$, which is generated during the combustion of natural gas containing $\mathrm{H}_{2} \mathrm{~S}$.

The removal of $\mathrm{CO}_{2}$ in a particular LNG plant is also aimed to avoid $\mathrm{CO}_{2}$ freezing that will plug the process unit in the liquefaction unit. Since the freezing point of $\mathrm{CO}_{2}$ is at $56.6^{\circ} \mathrm{C}$, the possible freezing could happen when the natural gas is liquefied at the temperature of minus $160^{\circ} \mathrm{C}$.

In industrial gas processing, there is an increasing interest in gas absorption processes for the selective removal of acid gases from the raw gas streams. The alkanolamine is a common chemical absorbent used in refineries to remove acid gases (Kohl and Riesenfeld, 1985). The alkanolamines of prime significance include monoethanolamine (MEA), diethanolamine (DEA), methyldiethanolamine (MDEA), diisopropanolamine (DIPA), and diglycolamine (DGA). The use of aqueous solutions of $N$-methyldiethanolamine (MDEA) to accomplish selective removal of acid gases was first proposed by Frazier and Kohl (1950). Besides MDEA, DIPA has also been reported to show a great selectivity for $\mathrm{H}_{2} \mathrm{~S}$ over $\mathrm{CO}_{2}$ than either MEA or DEA. DIPA has been used in the commercial Adip process and as a constituent of the Sulfinol process (Maddox, 1974; Maddox and Morgan, 1998; Ratman, 2002).

In general, the amine processes involves a few cycles of absorption and desorption in order to permit the use of the absorbent. Due to the closed loop nature of these processes, non-regenerable contaminants tend to accumulate and can cause major reduction in efficiencies and operational problems. The problem was related to the interfacial phenomena, which has to be understood in order to study the interaction of the undesired foam present during the counter current with the sour hydrocarbon-riched gas stream and the absorption solution of aqueous alkanolamines. Foam consists of gas bubbles dispersed in a liquid medium. Gravity encourages the liquid layer between the bubbles to drain and form the lamellar and plateau border regions. Liquid from the lamellar region drains toward the plateau border region, due to a pressure differences and the bulk viscous drag force, the surfactant surface concentration in the plateau border to be higher than that in the lamellar region adjacent to the bubble surface (Alargova et al., 2004).

The presence of a surface tension gradient on a bubble surface results in the spreading of surfactant molecules from regions of low surface tension to regions of high surface tension. This surface spreading process causes movement of the underlying layer of liquid in the direction opposite to that of liquid drainage, resulting in retardation of the liquid drainage and provision of transient stability to the foam (Bikerman, 1973). Few results have been reported on the foaming tendency of aqueous solutions of one alkanolamine: for 30 mass \% of DEA (McCarthy and Trebble, 1996) and 50 mass \% of MDEA (Yanicki and Trebble, 2006) with different contaminants in contact with nitrogen, methane, and ethane, at several temperatures; for MDEA and DEA in the range 0.2- 
4M in contact with nitrogen (Hesselink and van Huuksloot, 1985).

So far, there is no extensive experimental data on the foaming behaviour of aqueous solutions of blends of piperazine and $\mathrm{N}$-methyldiethanolamine on the impurities of natural gas found in the literature. Contaminants or impurities in amine solutions can arise from various sources and usually exist in several different forms. Although a single contaminant may necessitate a certain plan of action, gas treating solutions rarely contain only one or two impurities. Instead, many different impurities exist in varying concentrations, in which many of them may show some adverse effects on the process.

Generally, the impurities in natural gas are hydrocarbon liquids, iron sulfide, sodium chloride, acetic acid, methanol and glycol. At the high pressure and low temperature prevailing in the absorption tower, heavy hydrocarbons and even some lower boiling constituents of the feed gas are dissolved in the amine solutions (Jou et al., 1996). Most of the hydrocarbons with low boiling point are flashed off in the flash drum or are removed in the stripping tower. However, the heavy hydrocarbons tend to stay in solution and pose another form of contamination in amine solutions. It certainly develops foam activity in the unit system or even stable foam on the top of absorber or regenerator column.

Non-volatile contaminants arise from diverse sources such as gas wells or make up water. Other common types of nonvolatile contaminants are particulates, which may be carried into the amine solution by the raw feed gas. Iron sulfide is very common but appears as undesirable substance due to its potential to stabilize foams or enhance foaming tendencies. Besides the source coming from the upstream operations, iron sulfide also could be formed due to the presence of sulfur component in the carbon steel environment. In the case of long term operations, iron ( $\mathrm{Fe}$ ) from the equipment material that the protective film scratched will react with sulfur to form iron sulfide deposit or fine particles. In the close loop of amine circulation, this iron sulfide will definitely increase foam activity of the solvent.

Solid contaminants of any type can decrease the efficiency of the absorber and stripper by plugging contactor trays, contactor packing's and process piping. The presence of sodium chloride in the natural gas treating is usually found when seawater is used as cooling medium. The introduction of sodium chloride may happen into the unit due to some tube leaks on the sea cooling water exchangers. In some cases, the leaks on the lean amine cooler that was placed in the low pressure layout can be found. The leaks could occur when carbon steel tubes could not sustain from corrosion during the operations. The seawater cooling with a slightly higher pressure was introduced into the exchangers that predominantly ingress into the solvent circulation loops when there is a small leak found in the exchangers. It could accumulate in the system and may trigger corrosion on the stainless steel material and increased the total dissolved solid in the amine solvent, which creates severe foaming.

Acetic acid maybe introduced into amine unit along with delivery gas from the upstream side due to the upstream corrosion inhibitor injection. This corrosion inhibitor agent sometime contains acetic acid and carries over into amine unit, which may create a foaming. Besides, the acetic acid could present from the wells as a part of contaminants coming out and it could not be treated in the upstream gas treating units. Therefore, it is found accumulated in the liquid slugs along the pipeline and collected in the slug catcher's area. At the time of operation failure in this area, some liquids would carry over into the inlet facilities of the acid gas removal unit. It would then accumulate in the amine unit which may create foaming problem in the long run operation.

The presence of methanol is obviously foreseen when this chemical is injected into the inlet facilities or in the gas treating to prohibit hydrate formation. When the liquid separator is under performance to drop liquid mist from this injection, a small amount of methanol can be carried over into the amine unit to create severe foaming. The more hydrate formation is detected, the more frequent methanol is injected and the more possible foaming is foreseen. The other amine solvent contaminant that could present in the gas treating unit, such as glycol, is usually used as gas dehydration in the up stream process to avoid any hydrate formation along the pipeline. When glycol is carried over into the amine unit, it may create foaming in the system.

Therefore, in this study, the physicochemical characterizations of aqueous solutions of the piperazine and MDEA blend in the natural gas impurities are investigated. In order to identify and understand the characteristic of the foam behavior, it has been experimentally determined the foam ability of the solutions by measuring the foam height. This parameter in turns indicates the foam ability as a result of the impurities present. In addition, the foam stability as a function of collapse time for the same aqueous solutions of piperazine and MDEA is also observed.

\section{A. Materials}

\section{EXPERIMENTAL}

The sample of MDEA was obtained from an activated MDEA manufacturer (Taminco of Belgium) with a purity of $99.9 \mathrm{~mol} \%$. Water was distilled and de-ionized. The blend of piperazine and MDEA were twice distilled under vacuum with a stream of nitrogen in order to remove traces of moisture and other impurities. The impurities involved on the testing were methanol, hydrocarbon liquids, polyethylene glycol, sodium chloride, iron sulfide and acetic acid. The pure nitrogen $\left(\mathrm{N}_{2}\right)$ gas was used in the foam formation testing as bubbling gas.

\section{B. Preparation of Solutions}

All the studied aqueous solutions of known concentration of alkanolamines were prepared by mass using a Sartorius 2006MP analytical balance whose precision and accuracy is $\pm 0.0001 \mathrm{~g}$. It should be noted that although for simplicity all the concentration values for the studied systems are reported throughout this work to the first decimal digit they have an uncertainty of \pm 0.002 mass $\%$.

\section{Foamability}

The foam was determined by employing the experimental device shown schematically in Fig. 1. It is an in-house-built all-glass dynamic foam-meter. The foaming tube (Fig. 1), which is made from heavy-wall borosilicate glass precision tube with a calibrated of $1000 \mathrm{ml}$, is vertically positioned and contains at the base a fine fritted glass. The test has to be carried out at $25{ }^{\circ} \mathrm{C}$. The foam formation using different concentration of blends of amine solutions was determined. 
The spherical diffuser stone was used in the testing of foam formation. Prior usage the alkanolamines, the diffuser stone was used in $150 \mathrm{ml}$ distilled water for at least one hour. 150 $\mathrm{ml}$ of the blends of piperazine and MDEA solution sample was poured into a $500 \mathrm{ml}$ measuring cylinder and the diffuser stone was introduced into the solution.

A constant nitrogen flow of $60 \mathrm{Nl} / \mathrm{h}$ is flowed through the diffuser stone into the solution for 5 minutes. When the 500 $\mathrm{ml}$ mark reached before 5 minutes of nitrogen bubbling, 500 $\mathrm{ml}$ was noted as experimental result for the foam height and the nitrogen flow was stopped. The foam break time was recorded when the original height of $150 \mathrm{ml}$ is reached. This time is called foam collapse time. After the third test has been carried out, nitrogen gas was bubbled for another 30 minutes through the diffuser stone into distilled water and the water was changed for 2-3 times to clean the diffuser stone from the sample solution. Fig.1 illustrates the set up used for the foamability testing.
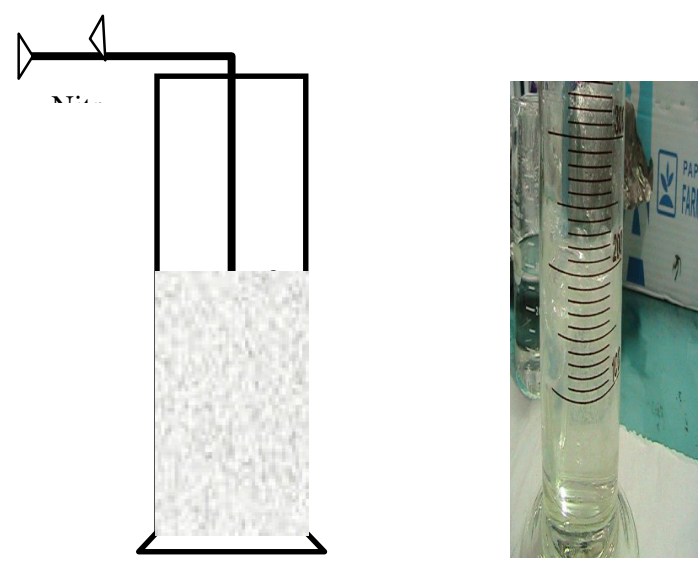

Fig. 1 Foamability test

\section{RESULTS AND DISCUSSION}

A. Foam Behaviour of Blends Piperazine-MDEA at Various Concentrations

In this study, the foam behaviour of aqueous solution of MDEA- piperazine blends are characterized in various concentrations and to identify the impact of the contaminant presences in the solutions as a function of type and concentration of impurities and alkanolamine solutions.

The foam behavior of various concentrations of piperazine-MDEA blend that have been subjected to the dilution with water would explain how the foaming tendency could be affected by the presence of water dilution. Water is a common dilution agent and it should not be regarded as contaminant. The presence of water is required to dilute the concentrated piperazine-MDEA to meet the specified amine solvent concentration during the acid gas removal unit operation. Moreover, the presence of water dilution could affect the foaming behavior of the amine solvent at the acceptable level.

The results of this study foam behavior could be used for column sizing design as called as foam factor. This parameter particularly influences the column tray spacing and down comer sizes. The foam behavior of the water diluted amine in various concentrations is shown in Fig. 2. As presented in Fig. 2, the blends of MDEA-piperazine are stabilized from foaming formation as indicated in the graph. The foam formation is negligible. Therefore, it can conclude that the foaming phenomenon can be avoided if the $\mathrm{CO}_{2}$ removal processes on the natural gas do not involve the gaseous impurities such as iron sulfide, methanol, organic acid and hydrocarbon.

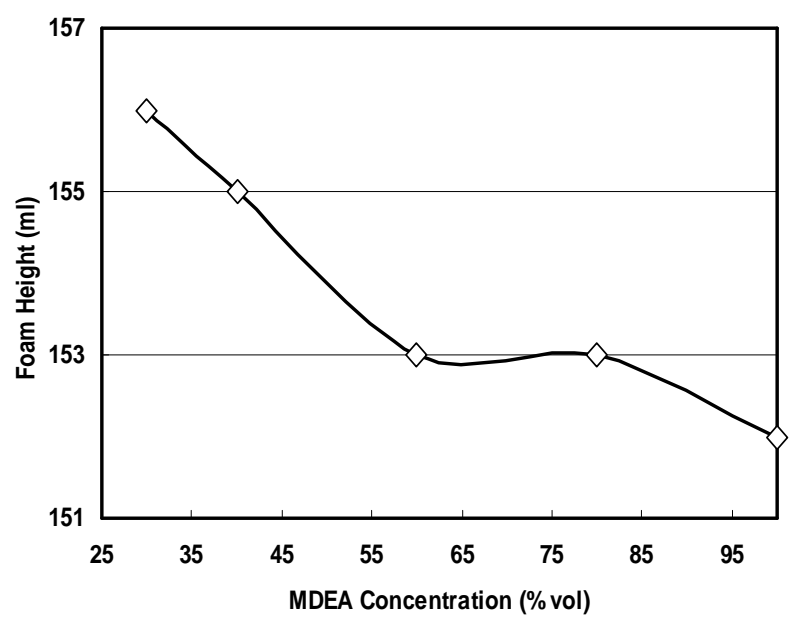

Fig. 2 Foam behaviour of MDEA-water system

\section{B. Foam Behaviour of Blends MDEA- Piperazine in the Presence of Contaminants}

The presence of contaminants might cause the blend of piperazine-MDEA to have excessive or stable foam. The effects of these contaminants at the various concentrations which have been diluted or dissolved into MDEA solvents as depicted in the Fig. 3 to 12 .

\section{1). Effect of Hydrocarbon on the Foam Formation}

The effect of hydrocarbon on the formation of foam on the solution of MDEA is shown in Fig. 3. Generally, hydrocarbons tend to stay in the solution and generate the foam in the amine solution (Jou et al., 1996). The concentration of hydrocarbon determined the foam formation in the solution. The foamability of aqueous solution of MDEA increased with the increasing concentration of impurities as evidenced in Fig. 3. As presented in Figure 4.3, firstly, the foam was formed on the concentration of MDEA about $30 \%$ and $5000 \mathrm{ppm}$ of hydrocarbon, respectively. Figure 4.3 also shows that the foamability can be reduced with increasing concentration of MDEA solution. The results indicated that the fresh of MDEA was difficult to form foam compared to other concentration. In other word, the fresh MDEA is more stable compared to other concentration of MDEA tested. It is because the presence of water as dilution agent will cause amine soap to form foam in the MDEA solution. Therefore, the fresh MDEA with the presence of hydrocarbon had low foam tendency. 




Fig. 3 Effect of different hydrocarbon concentration on the foam formation

\section{2). Effect of Iron Sulfide on Foam Formation}

Fig. 4 demonstrates the effect of iron sulfide as an impurity on the foam formation. Generally, iron sulfide can react with the water to form $\mathrm{Fe}(\mathrm{OH})_{2}$. Therefore, the presence of iron sulfide on the MDEA solution may lead to the foam formation on the solution. The formation of the oxide film in an aqueous system has been proposed as a series of anodic reactions involving adsorbed complexes:

$$
\begin{array}{lll}
\mathrm{Fe}+\mathrm{H}_{2} \mathrm{O} & \longrightarrow \mathrm{Fe}(\mathrm{OH})+\mathrm{H}^{+} \\
\mathrm{Fe}(\mathrm{OH}) & \longrightarrow \mathrm{FeO}+\mathrm{H}^{+} \\
\mathrm{FeO} & \longrightarrow \mathrm{Fe}_{2} \mathrm{O}_{3}+\mathrm{H}^{+}
\end{array}
$$

The molecules of $\mathrm{Fe}(\mathrm{OH})$ as shown on the reaction above will increase the foam formation in the solution of MDEA (Veldman, 2000). The foam consists of bubbles that dispersed in a liquid medium. As a bubble detaches from the spherical diffuser stone at the bottom of the column, it rises to the gasliquid interface, because its density is lower than that of the liquid phase. During the process, surfactant molecules such as iron sulfide in the liquid adsorb onto the bubble surface. Due to the hydrodynamic effect, the differences in surfactant coverage at the top and at the rear of a bubble may occur as a bubble rises. After reaching the gas-liquid interface, the bubble continues to travel through the foam phase as its size increase and bursts as it reaches the top of the foam phase.

The growth of bubbles in the foam can occur as a result of bubble coalescence or gas diffusion through the lamellae from smaller to larger bubble (Tan et al., 2005). In this mechanism, the surfactant molecules adsorb on the smaller bubbles is returned directly to the solution as the bubble collapse. As the consequence, the increasing concentration of iron sulfide in the solution of MDEA will give rise to the formation of foam as presented in Figure 4.4. The foam formation in blends solution of MDEA with iron sulfide as impurity was quite similar with hydrocarbon as impurity. This phenomenon indicated the same mechanism of growth of the bubble in the iron sulfide and hydrocarbon. However, at the same concentration, the foam height of iron sulfide is higher than that of hydrocarbon as impurity. Meanwhile, the foaming tendency has also not occurred in the fresh MDEA as depicted in the Fig. 4. This phenomenon has proved that the fresh of MDEA was also stable in the iron sulfide as impurity in the MDEA solution.

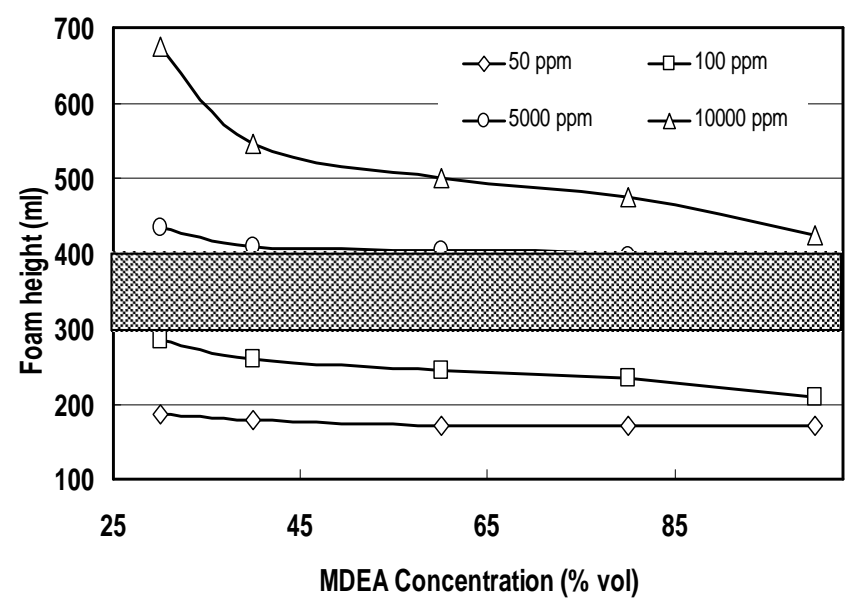

Fig. 4 Effect of different iron sulphide concentration on the foam formation

\section{3). Effect of Sodium Chloride ( $\mathrm{NaCl}$ ) on Foam Formation}

Fig. 5 displays the effect of $\mathrm{NaCl}$ on the foam formation onto aqueous solution of MDEA. $\mathrm{NaCl}$ can be dissolved in the MDEA solution and would reduce the MDEA quality. Moreover, in the solution of MDEA, sodium $\mathrm{NaCl}$ will form crystal and attach to air bubble (Aguila-Hernández, 2001). The attached sodium particles will form a network structure on the surface of the air bubble due to the particle-particle and particle-water interactions (Vijayaraghavan et al., 2006) in which finally the crystal of $\mathrm{NaCl}$ will lead to the foam formation.

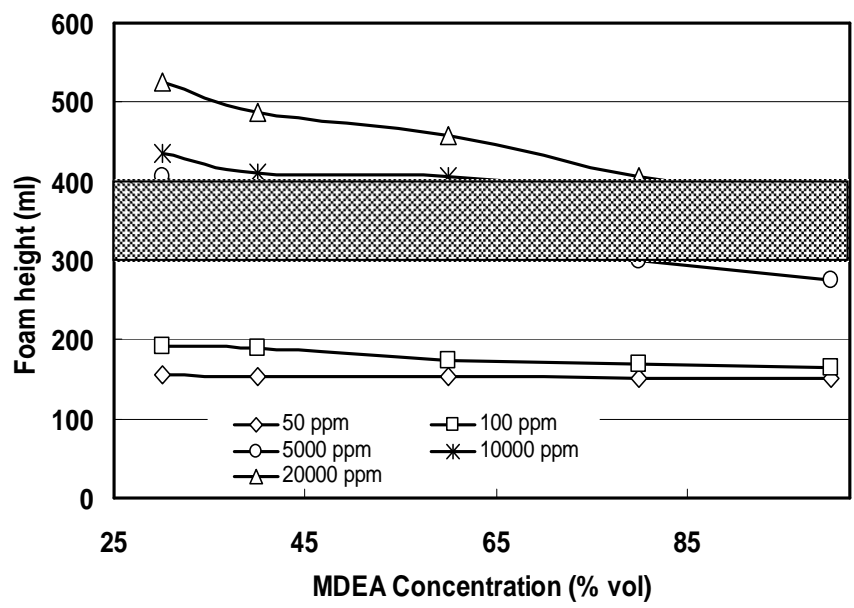

Fig. 5 Effect of different $\mathrm{NaCl}$ concentration on the foam formation

\section{4). Effect of Acetic Acid, Methanol, and Polyethylene} Glycol on the Foam Formation

Fig. 6 to 8 represents the effect of acetic acid methanol and polyethylene glycol on the foam formation in the blend of piperazine-MDEA solution. The results in Fig. 6 to 8 indicated that the type of impurities will determine the foam behavior of blend piperazine-MDEA. As shown in Figure 4.6, the foam height for acetic acid with concentration below $100 \mathrm{ppm}$ is far below the normal foam height of 300-400 ml for all MDEA concentrations. However, when acetic acid with concentration above 1000 ppm was used, the foam height reached above 400 $\mathrm{ml}$ for concentration of the solution of piperazine-MDEA.

This phenomenon indicated that the concentration of acetic acid above $5000 \mathrm{ppm}$ will raise foaming phenomenon in the 
solution of piperazine-MDEA blends. Meanwhile, only $30 \%$ MDEA with the acetic acid concentration about $1000 \mathrm{ppm}$ will significantly show high foaming tendency. As can be seen in Fig. 7 to 8 , for both with the impurities of methanol and polyethylene glycol, the foaming only occurred at the 30 $\%$ of MDEA. The decreasing foaming phenomenon in the methanol and polyethylene glycol might be due to the large particle of methanol and polyethylene glycol. Larger size particle cannot attach to the surface of the air bubble and prevent the bubble from approaching each other. This has caused a decrease in the foam formation which was also reported elsewhere (Dickinson et al., 2004).

5). Effect of Types of Impurities on the Foam Formation and Collapse Time of Foam

The effect of various impurities on the foam formation and collapse time is presented in Fig. 9 to 12. As shown in Fig. 9 and 11, the presence of iron sulfide in MDEA solution has contributed to the higher foam formation in the MDEApiperazine solution. The concentration of iron sulfide in the blend of solution MDEA-piperazine that reached up to 10,000 ppm has caused foam formation in all the MDEA concentration. Fig. 10 and 12 also indicate that iron sulfide was the main factor to affect the foam formation in the solution of blend MDEA-piperazine.

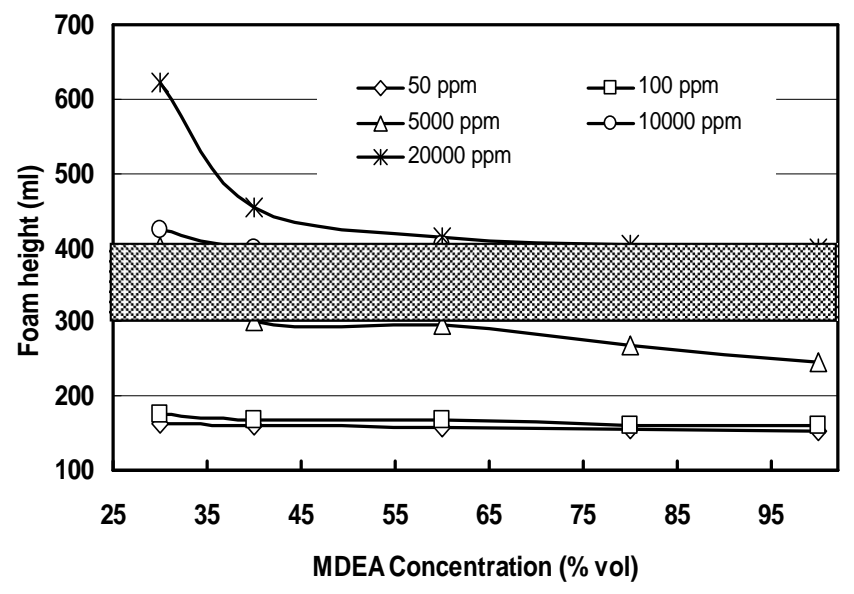

Fig. 6 Effect of different acetic acid concentration on the foam formation

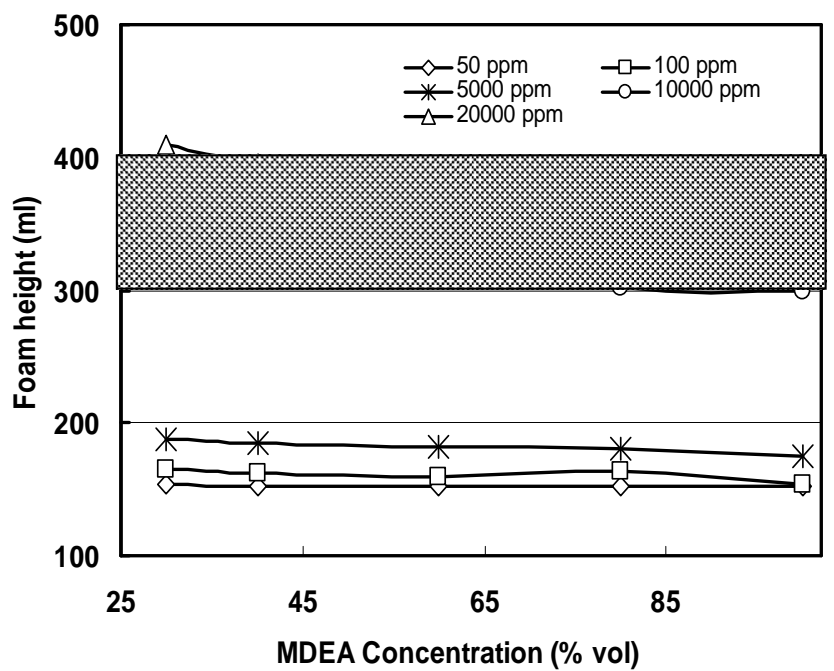

Fig. 7 Effect of different methanol concentration on the foam formation

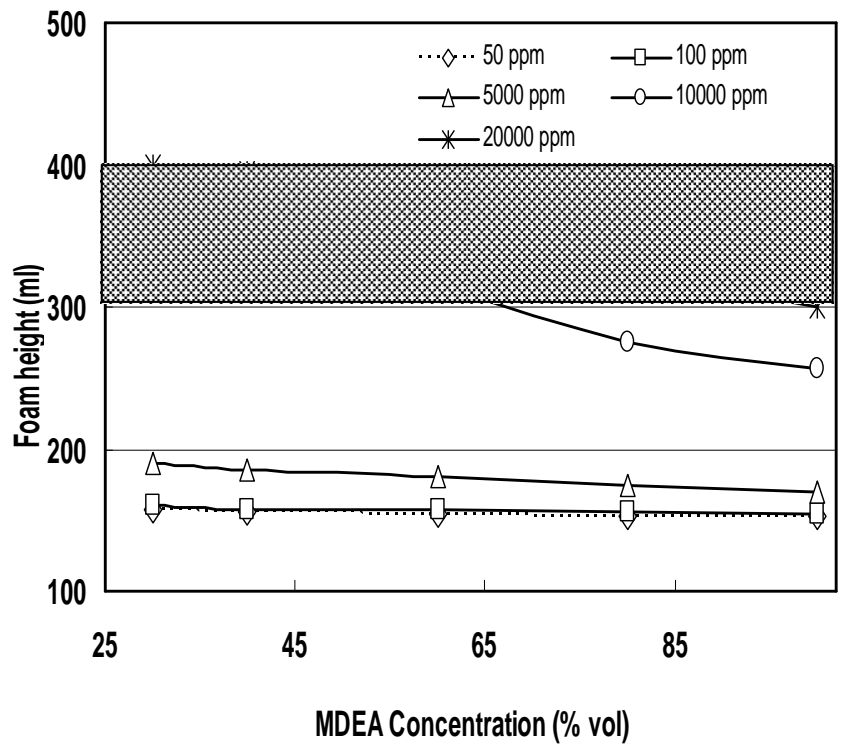

Fig. 8 Effect of different polyethylene glycol concentration on the foam formation

As depicted in Fig. 12, the collapse time of foam of the iron sulfide increased with the decreasing concentration of MDEA-piperazine. It was observed that the foam formed in the $10,000 \mathrm{ppm}$ of iron sulfide was stable and could be observed as permanent foam. Therefore, the presence of iron sulfide must be removed to prevent the foam formation. The Fig. 9 to 12 also show that the collapse time of foam formed of the hydrocarbon was not stable. However, the presence of the $\mathrm{NaCl}$ was found to cause the formation of permanent foam.



Fig. 9 Effect of types of impurities at 5,000 ppm on the foam formation 


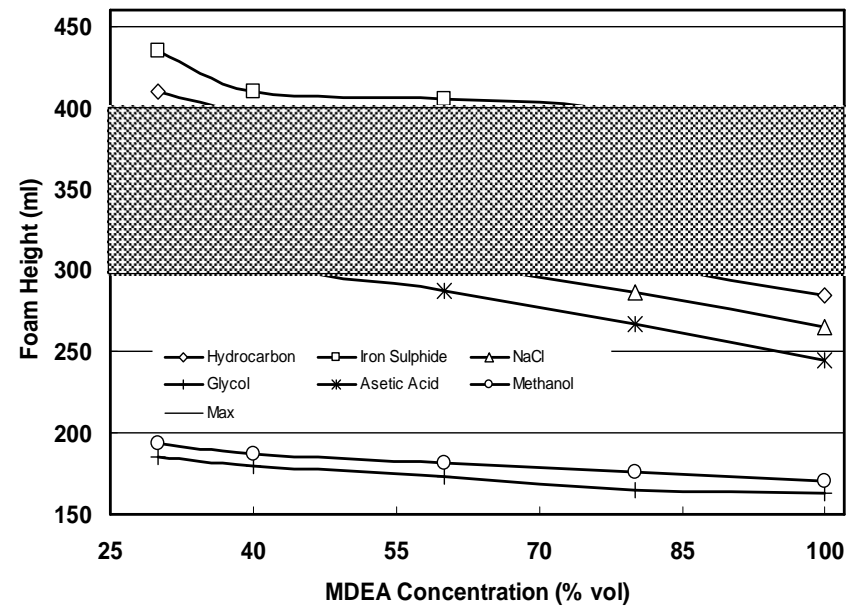

Fig. 10 Effect of types of impurities at 5,000 ppm on the collapse time

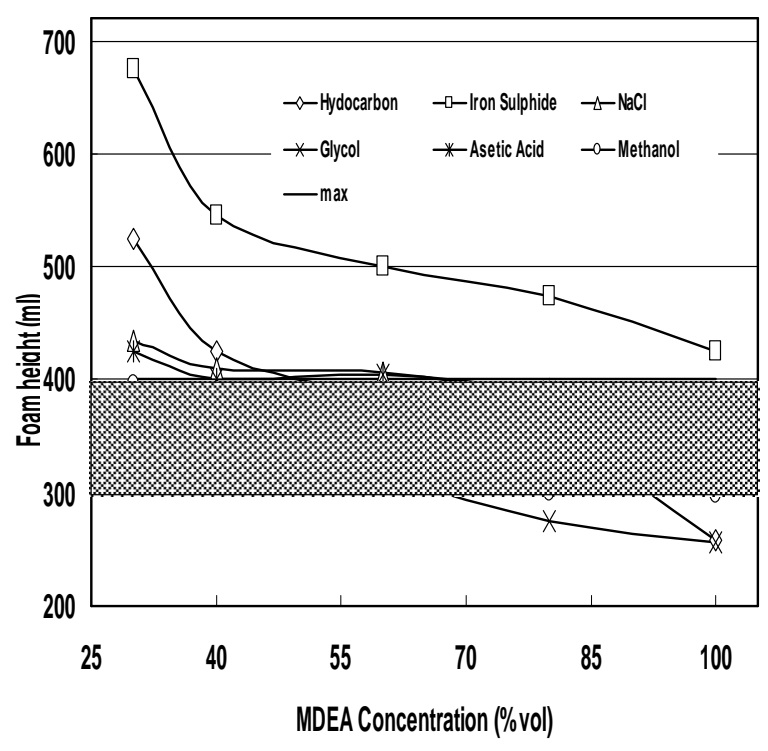

Fig. 11 Effect of types of impurities at $10,000 \mathrm{ppm}$ on the foam formation

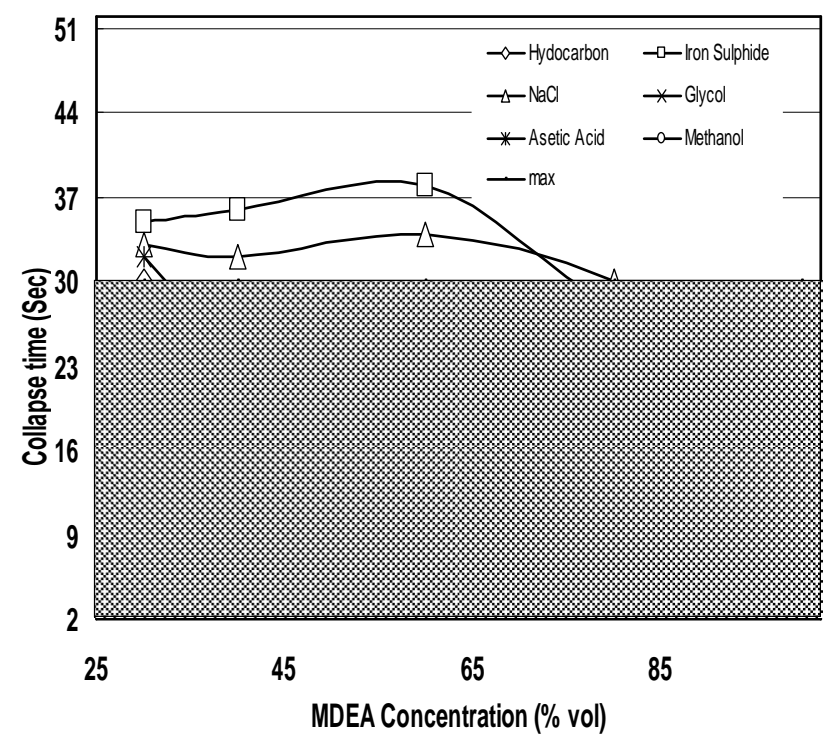

Fig. 12 Effect of types of impurities at $10,000 \mathrm{ppm}$ on the collapse time

\section{CONCLUSION}

A clear relationship was established between the impurities and foam behavior of blend solution of piperazine-MDEA. It was shown that the type of impurities and concentration of impurities have significantly affected the formation of foam. The concentration of MDEA has also significantly influenced the height of foam on the solution. Iron sulfide, hydrocarbon and sodium chloride are the impurities which apparently contributed to the high foaming tendency of the solutions. At the same concentration of the impurities, iron sulfide appeared as the most influential contaminant to the foam formation, which promoted the highest foamability in any concentrations of the blend piperazine-MDEA.

\section{REFERENCES}

[1]. Aguila-Hernández, J., Trejo, A,. Gracia-Fadrique, J., (2000) Surface Tension of Aqueous Solutions of Alkanolamines: Single Amines, Blended Amines and Systems with Nonionic Surfactants, Proceeding Fourteenth Symposium on Thermophysical Properties, June 25-30, Boulder, Colrado, U.S.A

[2]. Aguila-Hernández, J., Trejo, A,. Gracia-Fadrique, J., (2001), Surface tension of Aqueous Solutions of Alkanolamines: Single Amines, Blended Amines and Systems with Nonionic Surfactants, Fluid Phase Equilibria 185: 165-175.

[3]. Alargova, R.G., Warhadpande, D.S. Paunov, V.N. Velev, O.D. (2004) Foam Super-Stabilization by Polymer Microrods, Langmuir 20: 1037110374.

[4]. Bhide, B.D., Voskericyan, A., Stem, S.A. (1998) Hybrid processes for the removal of acid gases from natural gas, Journal of Membrane Science 140: 27-49

[5]. Bikerman, J.J. (1973), Foams, Springer-Verlag, New York.

[6]. Dickinson, E., Ettelaie, R., Kostakis, T., Murray, B.S., (2004), Factors Controlling the Formation and Stability of Air Bubbles Stabilized by Partially Hydrophobic Silica Nanoparticles, Langmuir 20: 8517-8525.

[7]. Hesselink, W.H. van Huuksloot, A. (1985), Foaming of Amine Solutions, Inst. Chem. Eng. Symp. Series 94: 193-202.

[8]. Jou, F.Y. Mather, A. E., and Otto, F. D., (1995), The Solubility of $\mathrm{CO}_{2}$ in a 30 Mass Percent Monoethanolamine Solution, Can J Chem Eng 73: $140-147$.

[9]. Koh., A.L. and Riesenfeld, F.C (1960), Gas Purification, $1^{\text {st }}$ Ed., McGraw-Hill.Gulf Publishing Company, Houston, Texas.

[10]. Maddox, R.N., Morgan, D.J. (1998), Gas Conditioning and Processing, Campbell Petroleum Series, Oklahama, USA.

[11]. McCarthy, J., Trebble, M.A., (1996), An Experimental Investigation into the Foaming Tendency of Diethanolamine Gas Sweetening Solutions, Chem. Eng. Commun. 144: 159-171.

[12]. Ratman, I (2002), BLNG Experience with SS-410 Tray material \& Shell snap-in valves", Distillation Meeting, Shell Global Solutions, Amsterdam

[13]. Tan, S.N. Fornasiero, D. Sedev, R. Ralston, J., (2005), Marangoni Effects in Aqueous Polypropylene Glycol Foams, Journal of Colloid and Interface Science 286: 719-729

[14]. Veldman, R.R., (2000), Alkanolamine Solution Corrosion Mechanisms and Inhibition From Heat Stable Salts and $\mathrm{CO}_{2}$ Corrosion, 00496

[15]. Yanicki, G., Trebble, M.A. (2006), Experimental Measurements of Foaming Tendencies in Aqueous Gas Sweetening Solutions Containing MDEA Over a Temperature Range of 297-358K and a Pressure Range of 101-500 kPa, Chem. Eng. Commun. 193: 1151-1163. 